\title{
Meeting Report: \\ Profiling of Drug Dissolution, Pharmacokinetics and Pharmacodynamics
}

Vivian A. Gray ${ }^{1}$ and Kim Huynh-Ba ${ }^{2}$

${ }^{1}$ Dissolution Technologies, Hockessin, DE, USA

${ }^{2}$ Pharmalytik, Newark, DE, USA e-mail:vagray@rcn.com
T he AAPS In Vitro Release and Dissolution Testing Focus Group cosponsored, along with the PROTEMP Group, a two-day workshop on Profiling of Drug Dissolution, Pharmacokinetics, and Pharmacodynamics, held in Kuala Lumpur, Malaysia, on September 2-3, 2015. The organizing institutions were the Non-Destructive Biomedical and Pharmaceutical Research Centre, iPROMISE, Universiti Teknologi MARA, and the University of Bath, UK. The goals and objectives of the workshop were:

- Share knowledge on critical relationships between dissolution, pharmacokinetics (PK), pharmacodynamics (PD), and in vitro-in vivo relationship analysis.

- Present Malaysia research activities and collaborations in dissolution, PK, and PD topics.

- Network for research collaboration, knowledge sharing, education, and industry exchange in dissolution, PK, and PD topics.

- Disseminate Malaysian regulatory information for dissolution testing.

- Provide information on dissolution method development, cGMP, and product stability issues.

Members of the Program Committee were Wong Tin Wui (Universiti Teknologi MARA), Nikoletta Fotaki (University of Bath), and Denise Ang (PROTEMP Group).

In their opening remarks, Nikoletta Fotaki and Wong Tin Wui reiterated the goals of the workshop and set the stage for the first session.

Sumitha Ganasegaram gave the first talk, titled "Regulatory Control of Generic Pharmaceuticals in Malaysia." She began by discussing the regulatory framework in Malaysia. The Control of Drugs and Cosmetic Regulation (CDCR) was established with Drug Control Authority (DCA) to regulate the pharmaceutical industry. The DCA is the executive committee responsible for product registration and licensing activities. The National Pharmaceutical Control Bureau (NPCB) carries out these activities. The Drug Registration Guidance Document (DRGD) is the parent guidance document to implement Malaysian regulatory requirements. She mentioned the Pharmaceutical Product Working Group established under the Association of Southeast Asian Nations (ASEAN) that has established many harmonization guidances adapted from the International Conference of Harmonization (ICH). She went on to describe the data requirements for generic pharmaceuticals. The efficacy data requirements include bioequivalence studies that follow two guidelines, the ASEAN Guideline for the Conduct of Bioavailability and Bioequivalence Studies and the FDA Guidance on the Biopharmaceutics Classification System. She described the challenges with generic approvals that include issues with comparators, biowaivers, and grandfathered products. She recommended an open dialogue and engagement with NPCB.

Sandra Klein (University of Greifswald) spoke on "Basic Principles of Compendial Dissolution Testing." She began by stating that dissolution testing is a very important tool in drug development and quality control. According to the United States Pharmacopeia, dissolution testing is required for all non-solution oral, including sublingual, pharmacopeial dosage forms in which absorption of the drug is necessary for the product to exert the desired therapeutic effect. Compendial dissolution testing should be conducted on equipment that conforms to pharmacopeial requirements. The choice of apparatus should be based on knowledge of the formulation and actual dosage form performance in the in vitro test system. This presentation gave detailed information on how to select the right test equipment, how to calibrate it, and how to avoid typical mistakes in routine dissolution testing.

The next speaker was Vivian Gray (V. A. Gray Consulting), whose talk was titled "Method Development and Setting Clinically Relevant Dissolution Specifications, Including Quality by Design." Her talk gave principles needed to 
develop a meaningful dissolution test, one that follows the concepts of $\mathrm{QbD}$. The emphasis was on understanding the product release mechanism and knowing the critical quality attributes, leading to clinically relevant dissolution specifications. The important aspects of method development were discussed in detail, including selection of filters, media, time points, volumes, apparatus, and other aspects of method development. The use of surfactants, sinkers, deaeration, and infinity points was discussed. An introduction to the attributes of a clinically relevant method along with design of experiments (DOE) was provided. The role of variability was discussed, and ways to minimize it were explored.

The afternoon session began with Vivian Gray giving an overview of the America Association of Pharmaceutical Scientists (AAPS), the U.S. organization that cosponsored the event. She highlighted the availability of e-membership for Malaysian scientists and the resources that AAPS offers.

The next speaker, Sandra Klein, spoke on "Designing Predictive Dissolution Test Methods for ImmediateRelease Formulations." She first asked if simulation of gastrointestinal conditions is essential to adequately predict the in vivo behavior of drug formulations. To reduce the size and number of human studies required to identify a drug product with appropriate performance, it would be advantageous to be able to prescreen formulations in vitro. For this purpose, the choice of both appropriate media and instrumental settings are crucial to forecast the in vivo performance of the test formulation. This presentation focused on how to develop predictive in vitro studies for oral IR formulations and gave an outlook on how to further improve the predictability of the in vivo performance of oral drug formulations with particular respect to various population subgroups.

Nikoletta Fotaki continued the program with "Designing Predictive Dissolution Test Methods for Modified-Release Formulations." She began by stating that the prediction of in vivo performance of oral modified-release (MR) dosage forms based on in vitro release testing is challenging. An in vitro release test based on physiological parameters and properties of the API and the MR dosage form can be used as a surrogate for the in vivo performance of the dosage form. The characteristics of release and the rationale on designing predictive in vitro release tests for MR formulations were discussed. Several case studies were presented.

Johannes Krämer (PHAST) presented the last talk of the session, "Interchangeability of Multisource
Products-How to Use In Vitro Methodology." This talk began with several definitions such as interchangeability, pharmaceutical equivalence, bioavailability, drug product performance, and bioequivalence. The experimental design of dissolution testing for product comparison was provided. In the case of BCS Class 2 and sometimes Class 4 drugs, dissolution may be considered the ratelimiting step for the bioavailability of the drug. Hence, a relationship between in vitro performance (i.e., dissolution) and in vivo performance may be established. In principle, this relationship is only valid for one particular product and one defined in vitro dissolution method including its specifications. This is commonly expressed as an in vitro-in vivo correlation (IVIVC). An example of in vitro and in vivo performance was discussed. He went on to show that BCS is linked to dissolution and absorption factors Dn, D0, and An. The challenge is to extend it to a group of products with generic properties. When the drug substance, its content, and the dosage form are equivalent, it is likely that the IVIVC can be extrapolated to more than one product. In those cases when the dosage forms are designed with different release-controlling mechanisms, the likelihood of a successful extrapolation is low. This applies to the so-called modified-release dosage forms, including delayed- and extended-release (in the EU: prolonged-release) dosage forms. He concluded by instructing that the USP General Chapter <1092> Dissolution Method Development and Validation as well as $<1088>$ In Vitro and In Vivo Evaluation of Dosage Forms provide guidance for pharmaceutical development.

On the second day, Johannes Krämer started the workshop with a presentation entitled "IVIVC According to USP General Chapter <1088> In Vitro and In Vivo Evaluation of Dosage Forms." Since the days of Gerhard Levy in the early 1960s, a relationship between in vitro performance and the corresponding in vivo performance of oral drug products for systemic action has been established in pharmaceutical science. In vitro dissolution has been considered a quality control tool to ascertain a required manufacturing quality. Johannes indicated that in vitro dissolution may also provide meaningful results on the in vivo performance of a certain product. This characteristic can be related to its bioavailability. As in clinical bioequivalence testing, in vitro performance testing may only prove similarity provided it has discriminatory power.

Nikoletta Fotaki gave the next talk on "Prediction of In Vivo Performance from In Vitro Dissolution Using In Silico Models." She noted that during drug development, approximately $40 \%$ of new compounds fail 
in the late clinical phases because of pharmacokinetics problems. Therefore, the successful prediction of human pharmacokinetics during preclinical research is of great importance. Recently, a physiologically based pharmacokinetic modeling (PBPK) approach has been used as a mechanistic quantitative platform for prediction of potential human absorption/bioavailability and for formulation development. Nikoletta discussed the use of biorelevant in vitro data within a physiologically based pharmacokinetic (PBPK) model environment for the prediction of in vivo performance and presented several case studies to demonstrate the predictability of the developed models.

Lo Yoke Lin (Universiti Malaya, Malaysia) presented the "Application of Population Pharmacokinetic Modeling in Clinical Trials." The study of pharmacokinetic and pharmacy-dynamic properties of a drug is an essential ingredient in the development of a drug and its clinical use. Plasma drug concentration variability among subjects when standard dosage regimens are administered is an important aspect in drug treatment. Population pharmacokinetics modeling and simulation assess the within-subject and between-subject pharmacokinetics variability. This population approach also helps to identify and quantify factors affecting the pharmacokinetics processes in a target population. The population-modeling approach has become a useful tool in all phases of clinical trials during drug development (Phase I, II, and III) as well as in clinical studies of patient populations where intensive blood sampling may be an issue; for instance, in young children including premature neonates. Indeed, a well-designed, executed, and evaluated population pharmacokinetics model can be used to save time and money by pooling and integrating all information collected from various clinical trials on the drug of interest.

Wong Tin Wui continued the workshop by presenting an interesting talk entitled "Development of Oral ColonSpecific Drug Delivery System-In Vitro Drug Release, In Vivo Pharmacokinetics and Pharmacodynamics Studies." Pectin is a natural heteropolysaccharide made of 1,4-linked $\alpha$-D-galactosyluronic acid residues and a range of neutral sugars such as rhamnose, galactose, arabinose, and lesser amounts of others. It is available in the form of free acid, simple salt such as sodium, potassium, and calcium salts, methyl ester, acetylester, feruloylester, or amidated polysaccharide. Pectin can form a gel matrix through $\mathrm{pH}$ and cationic cross-linkage modulation; therefore, it is useful as a colon-specific drug delivery vehicle. The polymer chains can be selectively digested by microflora in the colon to elicit site-specific drug release. It has the potential to keep the colon healthy, namely in the prevention of colon cancer. Colon cancer refers to cancerous growth in colon, rectum, or cecum. Typical chemotherapy is provided by the injection route to reduce tumor growth and metastasis. Recent research investigated the feasibility of oral delivery of chemotherapeutic agents. In comparison to injection, oral administration of drugs in the form of a colon-specific delivery system is expected to increase drug bioavailability at the target site and reduce drug dose and systemic adverse effects. He reviewed the physicochemical attributes of formulation/conjugation needed to retard drug release from the pectin matrix prior to its arrival at the colon and evaluated the therapeutic value of pectin in association with colon cancer. A multi-particulate pectin matrix is an ideal carrier to orally deliver drugs for the sitespecific treatment of colon cancer. Such a carrier has a slower transit and a higher contact time for drug action in the colon than a single-unit dosage form. Tin Wui also highlighted the recent approach where an in vivo coating was adopted to equip the multi-particulate pectin matrix with colon-specific drug release characteristics and the critical relationship between drug release, pharmacokinetics, and pharmacodynamics.

In the afternoon, Kim Huynh-Ba (Pharmalytik) discussed the "Regulatory Requirements of Stability Testing for Pharmaceutical Products." Although, the attendees were mainly clinical professionals, many have encountered GMP issues as stability data are obtained to determine the shelf life of the pharmaceutical product as well as establish the product specifications. Therefore, a sustainable stability program is critical to new pharmaceutical product registration. She discussed the ASEAN, WHO, and $\mathrm{ICH}$ stability requirements in designing a global program that can expedite a new product application. The emphasis is based on multisource/generic products. She also discussed climatic-zone, stress-study, and photo-stability requirements to support establishment of a stability profile of the product.

Vivian Gray continued the program speaking on "Gelatin Capsules: Current Issues When Cross-Linking Is Observed Related to the Addition of Enzyme to Media." Her presentation discussed the history behind the allowance of the use of enzymes in the dissolution testing of gelatin capsules and gelatin-coated tablets (sometimes known as Two-Tier testing). The current instructions in the USP General Chapter $<711>$ Dissolution are not very clear and pose some challenges; therefore, the chapter is being revised. The new revision includes the use of enzymes, 
pepsin, and surfactants that denature the enzyme. She also pointed out that this revision may not be harmonized with the European and Japanese Pharmacopoeia. She further explained that the USP General Chapter <1094> Capsules: Dissolution Testing and Related Quality Attributes became official on August 1, 2014, in the First Supplement to USP 37. Further changes are anticipated, and implementation processes will be discussed.

At the close of the workshop, Kim Huynh-Ba presented "Key Factors to Effectively Comply with Current Good Manufacturing Practices (GMPs)." GMPs are mandatory in the manufacturing of pharmaceutical products. She discussed critical GMP regulations and key factors to understand current compliance issues in the pharmaceutical industry. The major focus was on laboratory issues and related quality systems that can have a positive or negative effect on an organization. She introduced selected critical GMP requirements and discussed issues that are caused by an ineffective training program. She also gave updates on key issues with method validation and new initiatives emphasizing lifecycle management for analytical procedures. She also discussed documentation system infractions and the generation and evaluation of data.

Vivian Gray closed the workshop with remarks of thanks to the Malaysian organizers and speakers and appreciation for the fine hospitality shown to the AAPS Focus Group speakers. She remarked on the high quality of the presentations and thanked the organizers for the excellent job. The participants were very enthusiastic and engaged in the discussion, which indicated the workshop was a success.

There were 63 attendees from nine countries: Malaysia, Libya, Japan, Singapore, Indonesia, Pakistan, Syria, Yemen, and Australia. There were 34 delegates from universities, 23 delegates from industry, and 6 from government.

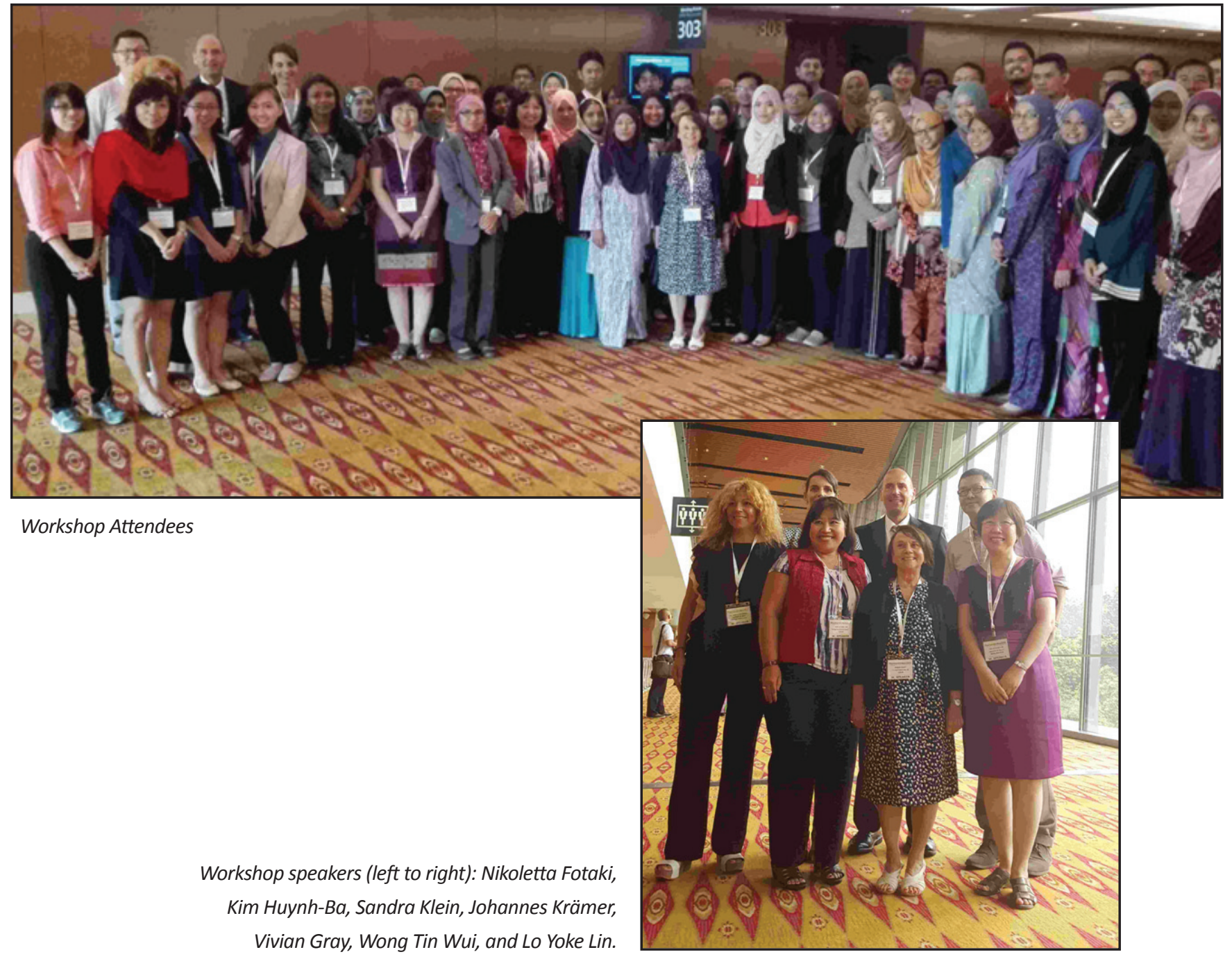

